\title{
A DFT Study on Electronic and Structural Properties of Graphene Nanoribbons
}

\author{
I.K. Petrushenko* \\ Physical and Technical Institute, Irkutsk National Research Technical University, 83, Lermontov st., \\ 664074, Irkutsk, Russia
}

(Received 22 August 2016; revised manuscript received 23 November 2016; published online 30 November 2016)

\begin{abstract}
We carry out quantum chemistry calculations on armchair graphene nanoribbons (AGNRs) using density-functional theory. PVE/SVP results for the dependence of energies of the highest occupied (HOMO) and lowest unoccupied molecular orbitals (LUMO) as well as energy gaps on AGNR widths and lengths are presented. We compare the HOMO and LUMO energies of a series of AGNRs with those energies of fullerene $\mathrm{C}_{60}$ and its soluble derivative, PCBM, to ascertain whether it is possible to use such AGNRs as electron acceptors in organic solar cells. The influence of Stone-Wales defects embedded in the frameworks of AGNRs on their electronic properties is also studied.
\end{abstract}

Keywords: Graphene nanoribbon, Electronic structure, DFT, Nanostructure, PBE.

DOI: $10.21272 /$ jnep.8(4(2)).04052

PACS numbers: 31.15.E -, 61.48. - c, 62.20.de, 71.15.Mb,

\section{INTRODUCTION}

Graphene, a perfect single layer of carbon atoms that are bonded together in a hexagonal honeycomb lattice, has become a focus of attention from both experimentalists and theorists since the discovery of Geim and Novoselov [1]. Unique properties of graphene predetermine its great influence on nanotechnologies [2-8]. However, the absence of a band gap of graphene sets the limits on the efficiency of graphene-based electronic and optoelectronic switching devices. Novel methods of a band gap opening are essential to advance its potential electronic applications. One effective way of creating a band gap in graphene is to confine charge carriers to a one-dimensional (1D) systems, namely graphene nanoribbons (GNRs), representing long stripes of graphene [9]. A variety of interesting edge and widthrelated phenomena emerge from the $1 \mathrm{D}$ nature of GNRs, whose properties depend considerably on their structures [10-12]. Thus, GNRs with armchair edges (AGNRs) are expected to exhibit width-dependent electronic band gaps, whereas zigzag GNRs (ZGNRs) are predicted to reveal spin-polarized edge states $[9,13]$. Recently, few effective methods have been proposed for the synthesis of GNRs [11,13-15]. Despite their fascinating properties, GNRs are not as popular as other carbon allotropes, fullerenes or single-walled carbon nanotubes (SWCNTs). For example, fullerene derivatives (for instance, phenyl- $\mathrm{C}_{61}$-butyric acid methyl ester or PCBM) are widely utilized in production of organic solar cells (OSCs) [16-18]. They used as electron acceptors in the process of exciton dissociation. To obtain effective charge separation and thereby high performance of OSCs, one should carefully select the relative arrangement of the LUMO levels of a donor and an acceptor. Bulky graphite and two-dimensional (2D) graphene are not suited for their employment as electron acceptors in OSCs. PCBM, in turn, has the strictly fixed LUMO level and good solubility, and it gives the best fit to the OSCs design. On the other hand, there exists a series of papers on structural and electronic properties of finite-length SWCNTs and GNRs. They showed that LUMO energies of SWCNTs and GNRs exhibit oscillating behavior on going from 'short' to 'long' models [19-22]. Thus, it is worth comparing electronic properties of PCBM and GNRs, and figure out whether the latter suitable for the effective separation of electrons and holes.

To the best of our knowledge, relatively low quantity of works is devoted to the investigations of the electronic properties of GNRs [9, 10, 23, 24]. Thus, for instance, the recent comprehensive work of $\mathrm{Wu}$ and Chai studied ZGNRs only [9]. However, one can definitely see that electronic properties of GNRs are strongly dependent on their edge shape. Therefore, here we selected finite-size AGNR models, because the case of short AGNRs is of special interest. It was already shown, when SWCNTs are shortened, their energy levels become quantized, which makes them suitable for various applications such as quantum dots and single-electron transistors [25]. From this point of view, it is very interesting to compare structural and electronic properties of finite-length SWCNTs and AGNRs.

Motivated by the above considerations, herein, we study structural and electronic properties of AGNRs of various lengths and widths by techniques based on density functional theory (DFT).

\section{COMPUTATIONAL METHODS}

In this paper, a series of polycyclic aromatic hydrocarbons represents finite-size AGNRs. The edge sites of AGNRs are saturated by hydrogen atoms to avoid the dangling. Fig. 1 represents a rectangular AGNR, with width $a$ and length $b$.

Thus, studied models are labeled as AGNR[a,b]. In particular, AGNR $[5,1]$ is a pentacene molecule. For all calculations, we used Orca 3.0.3 program package [27] employing the DFT method with the PBE functional $[28,29]$. The PBE has been demonstrated to be a reliable

\footnotetext{
*igor.petrushenko@istu.edu
} 


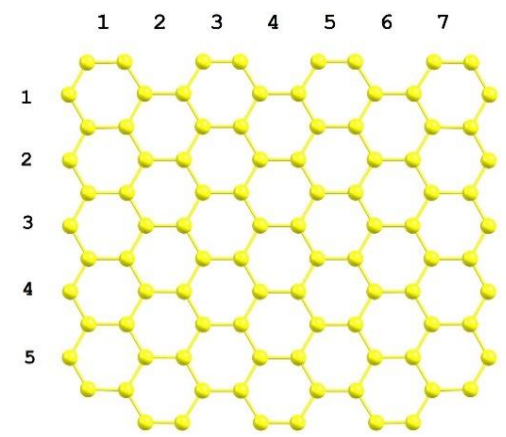

Fig. 1 - Representative model of AGNR[5,7], where width $=5$, length $=7$. Hydrogen atoms omitted for clarity

and commonly used functional for study of different nanostructures [30,31]. To calculate equilibrium geometries of the studied structures, we used SVP (split valence polarization) basis set, which is equivalent to the commonly used 6-31G* basis set [32]. In SVP the inner shell atomic orbitals are described by a single basis function, and two basis functions are provided for each valence shell atomic orbital, augmented by a set of polarization functions.

\section{RESULTS AND DISCUSSIONS}

Now we present theoretical predictions for the HOMO and LUMO energies of studied AGNRs as a function of the nanoribbon length (Fig. 2).

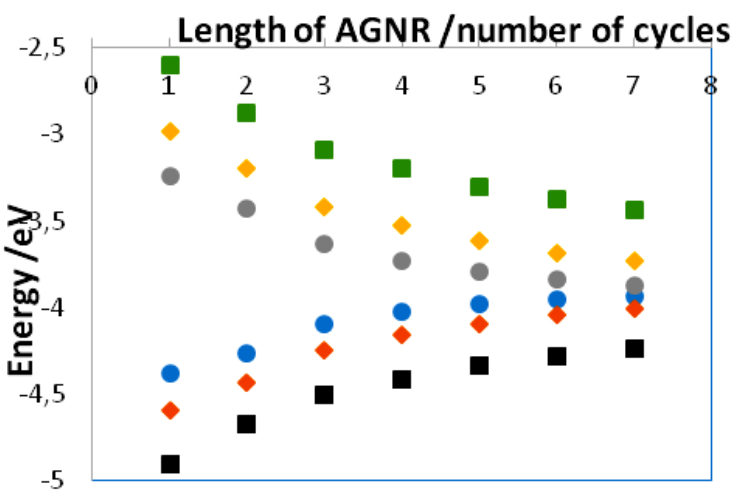

Fig. 2 - HOMO and LUMO energies (eV) vs. AGNRs length. AGNR [3, 7] (๘, HOMO (black), LUMO (green)), AGNR [4, 7] ( , HOMO (orange), LUMO (yellow)), AGNR[5, 7] (•, HOMO (light-blue), LUMO (grey))

For all considered AGNRs, we can see the monotonic increase in the HOMO energy with increasing AGNR length for all studied models. The wider models AGNR $[5, \mathrm{~b}]$ exhibit the lowest absolute value of HOMO and the highest of LUMO energy among AGNRs studied. This is not surprising, because such an AGNR, consisting of more quantity of carbon atoms, resembles properties of real 2D graphene better. In turn, AGNR $[4, \mathrm{~b}]$ shows intermediate values of the HOMO and LUMO, and AGNR $[3, \mathrm{~b}]$ exhibit the maximum gap between the HOMO and LUMO ( $\left.E_{g}\right)$ (Fig. 2). For any AGNR, energy values of monotonically increasing HOMOs and decreasing LUMOs yield a deceasing $E_{g}$ (it is roughly equal to the band gap in semiconductors). It should be noted, that the most interesting range of $E_{g}$ for nanophotonics is $\sim 1-3 \mathrm{eV}$, therefore the short AGNRs, 1-7 carbon rings long, are suited well for the design of promising AGNR-based application. Also, the change of the lengths of nanoribbons can be used to 'fine-tune' the electronic properties of AGNRs. Having analyzed Fig. 2 and Fig. 3, we may conclude that all studied AGNRs exhibit semiconducting properties.

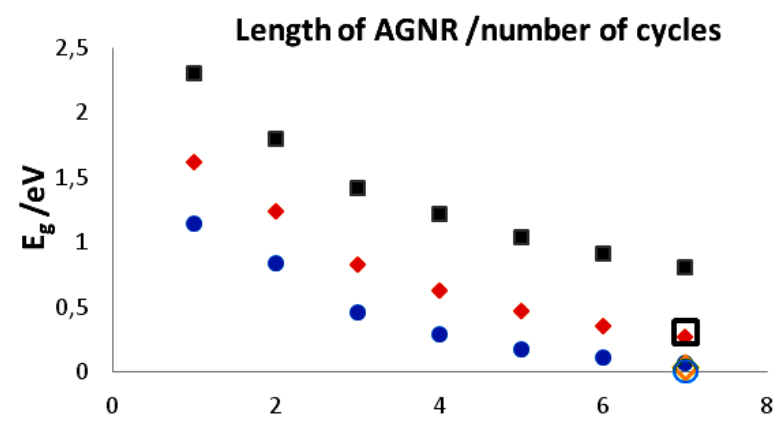

Fig. $3-E_{g}(\mathrm{eV})$ for studied AGNRs vs. their lengths. AGNR $[3, b](\bullet), \operatorname{AGNR}[4, b](\bullet), \operatorname{AGNR}[5, b](\bullet), \quad(b=1-7)$. The unfilled enlarged symbols denote $E_{g}$ values for AGNRs with the Stone-Wales defect (SWD) [ $a, 7],(a=3-7)$

AGNR $\left[3\right.$, b] are characterized by larger $E_{g}$ among all studied models. Clear tendency of $E_{g}$ to diminish on going from AGNR [a, 1] to AGNR [a, 7] $(a=3-5)$ reflects the fact of the energy gap disappearing in the case of 2D graphene (Fig. 3). The comparison of the present results with the Owens' work shows the same trends: gradual decrease in $E_{g}$ as the length of the ribbon increases; ionization potential that follows the HOMO energy according to Koopmans' theorem also diminishes with the increasing length of AGNR [23].

It is interesting to compare electronic properties of AGNRs with those properties of fullerene $\mathrm{C}_{60}$ and its soluble derivative, PCBM ([6,6]-phenyl- $\mathrm{C}_{61}$-butyric acid methyl ester), that are widely used for the design of novel OSCs. The substitution of the commonly used electron acceptor may lead to the increase in efficiency of such types of OSCs as the energy levels of AGNRs can be 'fine-tuned' by the alternation of their lengths.

According to recent literature data, $\mathrm{C}_{60}$ and $\mathrm{PCBM}$ have the following energetic properties: -6.400 (HO$\mathrm{MO}$ ), - 3.661 (LUMO), and $2.739\left(E_{g}\right) \mathrm{eV} ;-6.047$ (HOMO), -3.503 (LUMO), and $2.544\left(E_{g}\right)$ eV, respectively [26]. On comparison of the shortest AGNRs among studied, i.e. polycyclic aromatic hydrocarbons, with $\mathrm{C}_{60}$ and PCBM it can be seen that $\mathrm{C}_{60}$ energy levels are sufficiently lower (HOMO) and slightly lower (LUMO) than the corresponding levels of AGNRs (Fig. 2). The same holds true for PCBM: lower mutual arrangement of the frontier energy levels in comparison with those of AGNRs. $E_{g}$ of AGNRs is smaller than that of fullerene counterparts on $\sim 1 \mathrm{eV}$ in the case of AGNR [4,1] and [5, 1] (Fig. 3). AGNR [3, 1] have roughly the same $E_{g}$ comparing with $\mathrm{C}_{60}$ and PCBM. AGNR [3, 2], [4, 2], and $[5,2]$ exhibit an increase in HOMO energies, and decrease in LUMO energies, yielding a reduction of $E_{g}$. Electronic properties of these AGNRs show the considerable difference with those of $\mathrm{C}_{60}$ and PCBM. Further elongation of AGNRs leads to the gradual increase in HOMO and decrease in LUMO energies. The longest AGNRs [3, 7], [4, 7], and, at a larger degree, [5, 7] resemble properties of $2 \mathrm{D}$ graphene, and it is of no use to 
employ them in pristine form as electron acceptors in the OSCs. These data witness that despite the considerable alternation in energies of the frontier orbitals, the present model AGNRs could not be effective equivalents of widely used fullerene derivatives to substitute them in the design of OSCs.
Thus, our results on AGNRs of various lengths and widths show that the values of HOMO and LUMO levels as well as $E_{g}$ are length- and width-dependent. However, the direct substitution of PCBM on short AGNRs does not provide the necessary level of power conversion, because of non-optimal mutual arrangement of the HOMO and LUMO energy levels.

Table 1 - Energies of HOMOs, LUMOs and $E_{g}(\mathrm{eV})$ for pristine and defective AGNRs $[a, 7],(a=1-3)$

\begin{tabular}{|c|c|c|c|c|c|c|c|c|c|}
\hline & \multicolumn{3}{|c|}{ AGNR $[3,7]$} & \multicolumn{3}{|c|}{ AGNR $[4,7]$} & \multicolumn{3}{|c|}{ AGNR $[5,7]$} \\
\hline & HOMO & LUMO & $E_{g}$ & HOMO & LUMO & $E_{g}$ & HOMO & LUMO & $E_{g}$ \\
\hline Pristine & -4.242 & -3.434 & 0.808 & -4.009 & -3.735 & 0.274 & -3.940 & -3.871 & 0.069 \\
\hline Defective & -3.986 & -3.670 & 0.316 & -3.927 & -3.890 & 0.037 & -3.906 & -3.898 & 0.008 \\
\hline
\end{tabular}

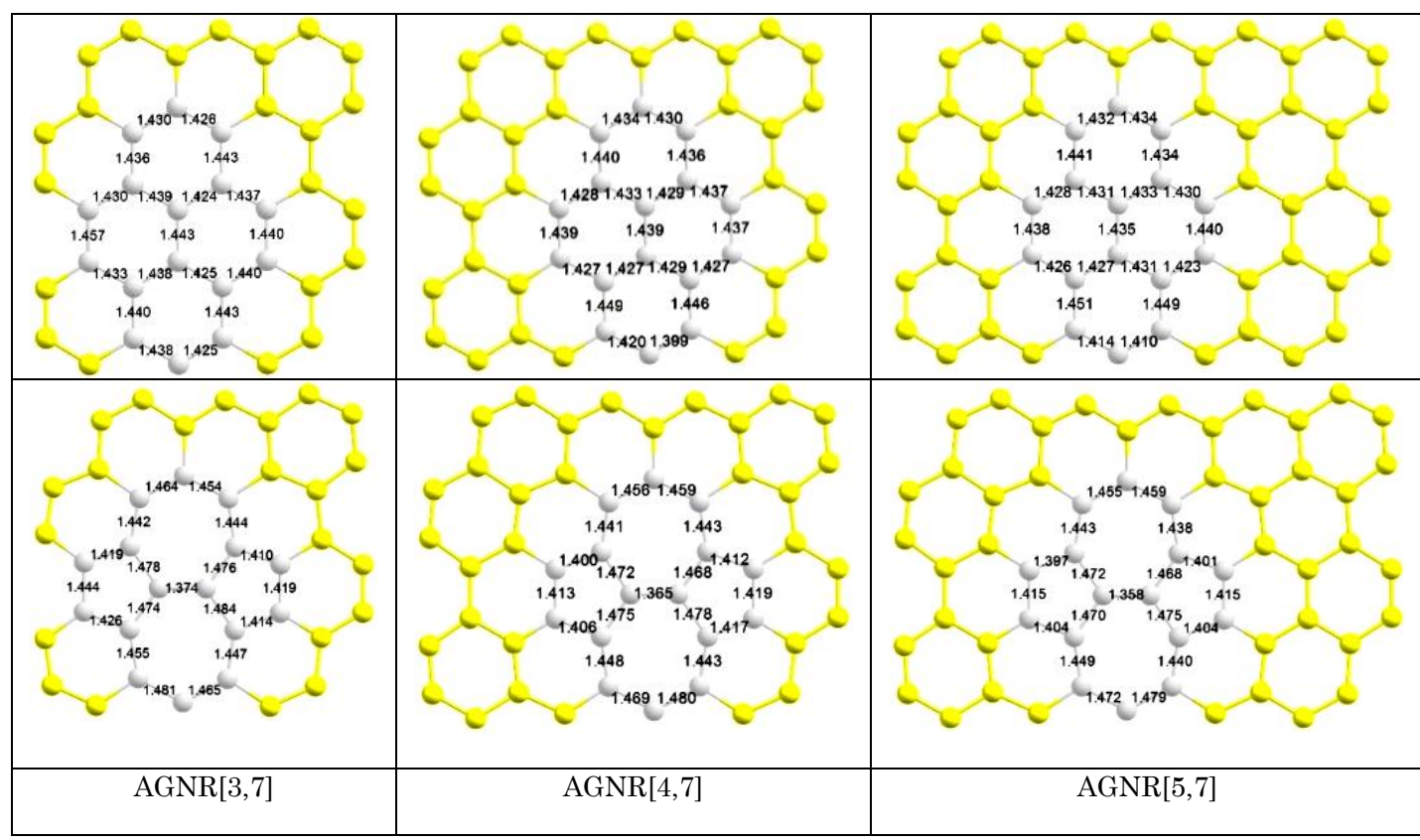

Fig. 4 - Fragments of surfaces of pristine (top) and defective (bottom) AGNR [3, 7], AGNR [4, 7], and AGNR [5, 7]. Hydrogen atoms omitted for clarity

At the same time, a careful adjustment of length can help researchers in obtaining AGNRs with 'fine-tuned' properties.

Next, we present theoretical predictions for electronic properties of the AGNRs with Stone-Wales defects (SWD) to check, whether their introducing can help scientists to alter electronic properties of AGNRs. A Stone-Wales defect is a defect that involves the $\mathrm{C}-\mathrm{C}$ bond rotation by $90^{\circ}$ with respect to the midpoint of the bond, yielding two five-membered and two sevenmembered cycles, instead of four adjacent six-membered cycles. AGNR [5,7] with one introduced SWD show moderate shifts in HOMO and LUMO energies $(\sim 0.03 \mathrm{eV}$ in each case), at the same time, $E_{g}$ decreases notably and the band gap becomes almost vanish $(0.008 \mathrm{eV})$ (Table 1 , Fig. 3). Defective AGNR [4, 7] exhibits the same trend, but the absolute values of changes increase: HOMO and LUMO energies are changed on introducing an SWD ( 0.08 and $0.15 \mathrm{eV}$, respectively), and its $E_{g}$ also decreases $(0.037 \mathrm{eV})$ (Table 1$)$.

The larger value of $E_{g}$ in the case of AGNR [4, 7] is the result of their larger deviation from $2 \mathrm{D}$ graphene. In the case of narrower AGNR [3, 7], we can see even more energy shifts: $\sim 0.25 \mathrm{eV}$ for HOMO and $0.24 \mathrm{eV}$ for LUMO. This leads to the substantial decrease in $E_{g}$, alt- hough it remains a non-zero value (0.316 eV) (Fig. 3).

To summarize the data obtained, the wider AGNR the better it resembles properties of real graphene, and vice versa the narrow AGNRs are similar to polycyclic aromatic hydrocarbons. At any rate, the introducing defects in AGNRs' structure can help one to modify their electronic properties. The remarkable case is defective AGNR [3,7], in which we can see considerable HOMO and LUMO shifts and the large $E_{g}$ drop. Such a scenario is caused by the relatively higher ratio of 'disturbed' to 'undisturbed' carbon atoms in comparison with defective AGNR [4, 7] and AGNR [5, 7].

To explain energy difference of frontier orbitals of defective and pristine AGNRs, we should involve into consideration C-C bond lengths adjacent to the SWD spot (Fig. 4). Four adjacent six-membered cycles consist of $19 \mathrm{C}-\mathrm{C}$ bonds (Fig. 4). Their length are approximately equal to $1.440 \AA$ for the bonds parallel to the direction of the AGNR axis (from the bottom to the top of the page), and to $1.434 \AA$ for the bonds diagonal to the direction of the axis. The latter tend to be elongated in the defected AGNR. As a result of a mutual redistribution, there arise novel C-C bonds, perpendicular to the AGNR axis. They are rather short with bond lengths of $\sim 1.360-1.370 \AA$. Also, rearrangements occur in hepta- 
gons and pentagons of the SWD, although it does not lead to outputs of these rings from the plane of AGNRs. Large C-C bonds alternations as well as reorganization of the surface of AGNR result in changes in electronic properties of AGNRs. The effect is more notable in the case of AGNR $[3,7]$ as the $\mathrm{SWD} /$ carbon atoms ratio is the largest among all studied nanoribbons. The orientation of the SWD on the AGNRs' surface also may leads to the alternation of their HOMO and LUMO energies.

Thus, the introducing of defects into AGNRs network can be a promising way to improve their electronic properties. The data obtained as well as recent successes in design of GNRs with 'atomic accuracy' allows us to expand our predictions to a practical side.

\section{CONCLUSION}

We have presented the DFT study on the electronic and structural properties of AGNRs. 21 AGNRs of different widths and lengths have been investigated: AGNR $[a, b],(a=3-5 ; b=1-7)$. HOMO and LUMO energies of all studied models exhibit the same trend: monotonic increase in the HOMO and corresponding decrease in the LUMO. The behavior of frontier orbitals of AGNRs and their structural analogues, SWCNTs, on elongation is opposite: the monotonic character alters on oscillations.

\section{REFERENCES}

1. K.S. Novoselov, A.K. Geim, S.V. Morozov, D. Jiang, Y. Zhang, S.V. Dubonos, I.V. Grigorieva, A.A. Firsov, Science 306, 666 (2004).

2. I.K. Petrushenko, J. Nano- Electron. Phys. 5 No 4, 04065 (2013).

3. X. Zhang, M. Han, S. Chen, L. Bao, L. Li, W. Xu, RSC Adv. 3, 17689 (2013).

4. I.K. Petrushenko, Monatsh. Chem. 145, 891 (2014).

5. S. Yadav, Z. Zhu, C.V. Singh, Int. J. Hydrogen Energy 39, 4981 (2014).

6. L. Liang, V. Meunier, J. Phys. Chem. C 119, 775 (2015).

7. M. Pykal, P. Jurecka, F. Karlicky, M. Otyepka, Phys. Chem. Chem. Phys. 18, 6351 (2016).

8. I.K. Petrushenko, Adv. Mater. Sci. Eng. 2015, 262513 (2015).

9. C.-S. Wu, J.-D. Chai, J. Chem. Theory Comput. 11, 2003 (2015).

10. M. Baldoni, A. Sgamellotti, F. Mercuri, Chem. Phys. Lett. 464, 202 (2008).

11. Y.C. Chen, D.G. de Oteyza, Z. Pedramrazi, C. Chen, F.R. Fischer, M.F. Crommie, ACS Nano 7, 6123 (2013).

12. L. Ma, J. Wang, F. Ding, ChemPhysChem 14, 47 (2013).

13. P. Ruffieux, S. Wang, B. Yang, C. Sanchez-Sanchez, J. Liu, T. Dienel, L. Talirz, P. Shinde, C.A. Pignedoli, D. Passerone, T. Dumslaff, X. Feng, K. Mullen, R. Fasel, Nature 531, 489 (2016).

14. J. Cai, P. Ruffieux, R. Jaafar, M. Bieri, T. Braun, S. Blankenburg, M. Muoth, A.P. Seitsonen, M. Saleh, X. Feng, K. Mullen, R. Fasel, Nature 466, 470 (2010).

15. P. Ruffieux, J. Cai, N.C. Plumb, L. Patthey, D. Prezzi, A. Ferretti, E. Molinari, X. Feng, K. Mullen, C.A. Pignedoli, R. Fasel, ACS Nano 6, 6930 (2012).
In terms of structure, the widest models used herein resemble properties of $2 \mathrm{D}$ graphene better, therefore they have the energetically close HOMO and LUMO and the smallest energy gap. AGNRs $[4, b]$ have intermediate values of HOMO and LUMO, and the narrow AGNRs $[3, b]$ exhibit the largest $E_{g}$.

In comparison with pristine fullerene, $\mathrm{C}_{60}$, and its soluble derivative, PCBM, AGNRs demonstrate such a mutual arrangement of frontier orbitals that cannot be effectively used in the design of OSCs. However, a convenient way to alter the electronic properties of AGNRs is their step-by-step elongation.

For all studied AGNRs, the introduction of SWDs into their frameworks moderately modifies HOMO and LUMO energies; at the same time, the energy gaps undergo substantial changes. The effects of the SWD defect are especially considerable in the case of the narrowest AGNR $[3, b]$. Our future studies will be devoted to investigations of electronic properties of a broader range of AGNRs both carbon and boron nitride.

\section{ACKNOWLEDGEMENTS}

IKP thanks Dr V. Malkin for his continued support and encouragement. IKP thanks SAIA for its support. The generous allotment of computational time from the Computer center of Novosibirsk State University (www.nusc.ru) is gratefully acknowledged.

16. M.C. Scharber, D. Mühlbacher, M. Koppe, P. Denk, C. Waldauf, A.J. Heeger, C.J. Brabec, Adv. Mater. 18, 789 (2006).

17. X. Liu, R. He, W. Shen, M. Li, J. Power Source. 245, 217 (2014).

18. H. Sahu, A.N. Panda, Macromolecules 46, 844 (2013).

19. I.K. Petrushenko, N.A. Ivanov, Physica E 54, 262 (2013).

20. I.K. Petrushenko, N.A. Ivanov, Mod. Phys. Lett. B 27, 1350210 (2013).

21. A. Galano, Chem. Phys. 327, 159 (2006).

22. Z. Zhou, M. Steigerwald, M. Hybertsen, L. Brus, R.A. Friesner, J. Am. Chem. Soc. 126, 3597 (2004).

23. F.J. Owens, J. Chem. Phys. 128, 194701 (2008).

24. M. Ghadiry, A.B.A. Manaf, M. Nadi, M. Rahmani, M.T. Ahmadi, J. Comput. Theor. Nanosci. 9, 2190 (2012).

25. A. Rochefort, D.R. Salahub, P. Avouris, J. Phys. Chem. B 103, 641 (1999).

26. Z. Mahdavifar, H. Salmanizadeh, J. Photochem. Photobiology A: Chem. 310, 9 (2015).

27. F. Neese, Wires Comput. Mol. Sci. 2, 73 (2012).

28. J.P. Perdew, K. Burke, M. Ernzerhof, Phys. Rev. Lett. 77, 3865 (1996).

29. J.P. Perdew, K. Burke, M. Ernzerhof, Phys. Rev. Lett. 78, 1396 (1997).

30. I.K. Petrushenko, K.B. Petrushenko Monatsh. Chem. 146, 1603 (2015)

31. M.G. Apenova, O.O. Semivrazhskaya, E.V. Borkovskaya, N.M. Belov, I.N. Ioffe, V.Y. Markov, S.I. Troyanov, N.S. Lukonina, L.N. Sidorov, A.A. Goryunkov, Chem. - An Asian J. 10, 1370 (2015).

32. H.H. Ansgar Schafer, R. Ahlrichs, J. Chem. Phys. 97, 2571 (1992). 